\title{
Antibiotics as food for bacteria
}

\section{Christopher J. Schofield}

Email christopher.schofield@chem.ox.ac.uk

1 Chemistry Research Laboratory University of Oxford Mansfield Road OxfordOX1 3TA UK

Antibiotic resistance against the penicillins and other $\beta$-lactams is of paramount therapeutic importance. Although penicillin resistance is known to involve degradation by hydrolysis, the molecular details of what happens next remain unclear. A new study reveals how soil microorganisms perform $\beta$-lactam catabolism and how this process can support bacterial growth.

Antibiotics are arguably the most important of small-molecule-based medicines. Like many other classes of antibiotics, $\beta$-lactams, such as the penicillins, have origins in natural products that are present in many soil bacteria. Soon after the mass introduction of antibiotics, it was recognized that in addition to their role as antimicrobials, they could be used as carbon and nitrogen sources by soil bacteria

[12]

. However, remarkably little has been reported on the biochemistry of antibiotic catabolism. In a recent Article, Crofts et al. report a conserved pathway for penicillin catabolism involving initial hydrolysis catalysed by $\beta$-lactamases - the same enzymes that are widely responsible for resistance to the penicillins and subsequent generations of $\beta$-lactam antibiotics

[3]

The penicillins were first introduced for clinical use more than 70 years ago. Despite the global spread of resistance, the penicillins and successive generations of $\beta$-lactams (including the cephalosporins and carbapenems) remain the most important class of antibacterials

[4]

. The $\beta$-lactams work by reacting with the nucleophilic serine of transpeptidase enzymes that are crucial for bacterial cell wall biosynthesis, forming a stable acyl-enzyme complex (Fig. 1). The most important mechanism of resistance to the $\beta$-lactams involves their hydrolysis as catalysed by $\beta$-lactamases

[5]

. The 'serine $\beta$-lactamases' are proposed to have evolved from the transpeptidases by learning how to hydrolyse the acyl-enzyme complex to form an active $\beta$-amino acid (penicilloic acid in the case of the penicillins) (Fig. 1).

Fig. 1 
Key elements of penicillin biosynthesis, industrial production, mode of action and catabolism.

Penicillin biosynthesis starts with the condensation of three amino acids ( $L-\alpha-$ aminoadipic acid, L-cysteine and L-valine) into a tripeptide, in a reaction catalysed by ACVS. The resultant tripeptide (ACV) is then converted into the bicyclic intermediate isopenicillin N by IPNS. Side-chain exchange is catalysed by an amidase/acyltransferase to give, for example, penicillin G. Related amidases are used industrially to convert penicillin $G$ to 'semi-synthetic' penicillins, such as ampicillin, via 6-aminopencillanic acid. The mode of action of penicillins and other $\beta$-lactams involves near irreversible acylation of transpeptidases involved in cell wall biosynthesis. By contrast, serine $\beta$ lactamases can efficiently hydrolyse analogous acyl-enzyme intermediates to give inactive penicilloic acids, for example, benzylpenicilloic acid, so enabling resistance. The same $\beta$-lactamases are involved in catabolism of penicillins in soil. Catabolic amidases, belonging to a different family to those involved in penicillin biosynthesis or industrial production, produce phenylacetic acid, which can then be used as a carbon source for bacterial growth. 


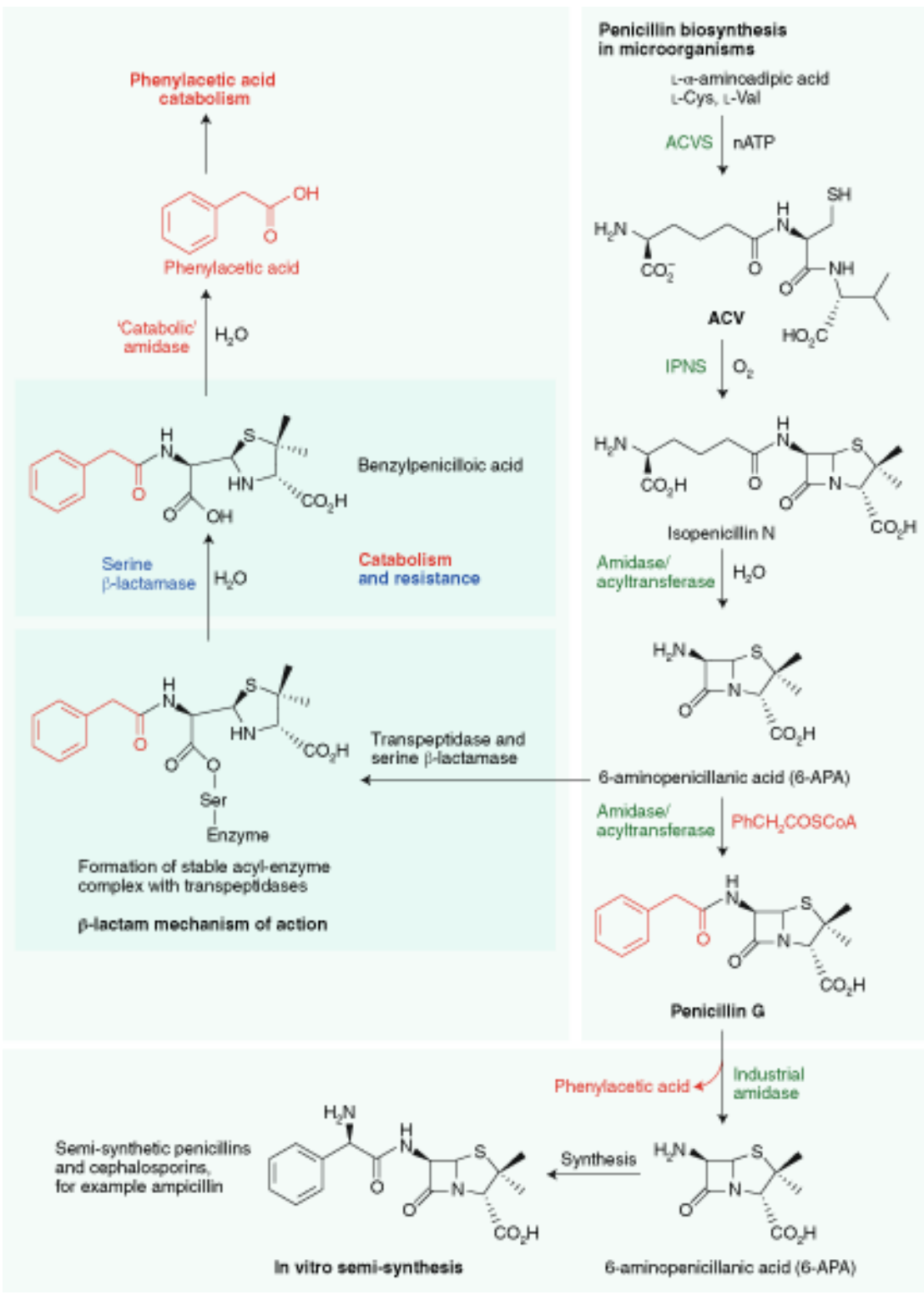

The penicillins are natural products, and clinically useful penicillins are produced by direct fermentation or modification of fermented materials, as in the case for the cephalosporins. Other classes of $\beta$-lactam antibacterials are produced by synthesis, such as the carbapenems and monobactams, but all have their origin in natural product structures. Penicillin biosynthesis proceeds via a common intermediate, isopenicillin N, which is produced in a paradigm of biosynthetic efficiency via two reactions catalysed in sequential fashion by the enzymes $\delta$-(L- $\alpha$-aminoadipyl)-L-cysteine-D-valine synthetase (ACVS) and isopenicillin N synthase (IPNS), with the latter step involving a remarkable bicyclization process

[6]

(Fig. 1). However, the C6 $\alpha$-aminoadipoyl side chain of isopenicillin $\mathrm{N}$ is not appropriate for clinical use. Hence, it can be 'swapped', either within microorganisms, or with isolated fermented penicillin products, to give clinically useful penicillins such as penicillin $\mathrm{G}$, which is produced by swapping with phenylacetic acid 
. Amidases, belonging to the $\mathrm{N}$-terminal nucleophilic class of hydrolytic enzymes, are involved in side-chain swapping in cells, and are extensively used on an industrial scale to catalyse hydrolysis of the C6-amide group of fermented penicillins to give 6 aminopenicilloic acid, which can be acylated to give clinically useful antibiotics (Fig. 1). The work of Crofts et al. sheds light on the mechanism by which proteobacteria in soil catabolise penicillins

. Following from work involving growth of four strains of proteobacteria using penicillin $\mathrm{G}$ as the carbon source, genome sequences of the organisms were obtained. The genomic analyses revealed the presence of genes encoding for multiple serine $\beta$-lactamases from all three classes previously implicated in $\beta$-lactam resistance (classes A, C and D), as well as genes encoding for enzymes of phenylacetic acid catabolism. The authors thus proposed that penicillin catabolism proceeds via initial $\beta$-lactamase-mediated hydrolysis to originate benzylpenicilloic acid, which is then converted to phenylacetic acid. In support of this hypothesis, transcriptomic analyses with a Pseudomonas strain grown on penicillin $\mathrm{G}$, or metabolites thereof, showed upregulation of genes encoding for a catabolic pathway in which hydrolysis catalysed by $\beta$-lactamases and amidase enables the production of phenylacetic acid, which can then undergo conversion into acetyl coenzyme A (CoA) and succinyl CoA, via established processes of phenylacetic acid catabolism (Fig. 1).

The assignment of the catabolic pathway was validated by engineering it into Escherichia coli, thereby enabling recombinant bacteria to survive on penicillin $\mathrm{G}$ as the sole carbon source. Notably, bacterial growth was supported by intact penicillin G and phenylacetic acid, but not by the 6-aminopencillanic acid penicillin core [3]

. The $\beta$-lactamases involved in penicillin catabolism appear to be the same, or closely related to those involved in antibiotic resistance, suggesting that $\beta$-lactamases may have functional roles in both catabolism and resistance. The results thus raise the interesting possibility that catabolic enzymes act as a reservoir of resistance-enabling enzymes, or vice versa. This concept of dual functionality may have consequences for 'fitness cost' calculations concerning drug resistance.

In contrast to the apparent conservation of $\beta$-lactamases in resistance and catabolic roles, the amidases involved in penicillin catabolism appear to belong to a different family of hydrolases to those involved in penicillin biosynthesis, and those which are used industrially for production of 6-amino penicillanic acid [3]

. The reason for this difference is unclear, but may reflect more than simple evolutionary chance. In light of the widespread use of antibiotics and their potential to serve as $\mathrm{C}$ and $\mathrm{N}$ sources for bacterial growth, the identification of pathways involved in their catabolism may be considered unsurprising. However, microorganisms were likely 
producing antibiotics long before human use, probably for hundreds of millions of years, and hence antibiotic catabolism may well have ancient origins.

Finally, the authors suggest the penicillin catabolism pathway might be used for bioremediation of antibiotic contaminated soil, though point out that such a use runs the risk of spreading resistance

[3]

. Whether or not this use is realised, the work highlights the importance of working on antibiotic resistance and metabolism within the natural context of soil. In this regard, the results raise interesting questions including what happens to the part of the penicillin molecule not producing phenylacetic acid that results from $\beta$-lactamase-mediated degradation in the soil (that is, the thiazolidine ring). Future studies may also elucidate the extent to which resistance enzymes also have 'moonlighting'

[8]

roles in antibiotic catabolism in other settings.

Competing interests

The author declares no competing interests.

References

1.

Kameda, Y. et al. Nature 191, 1122-1123 (1961).

2 .

Abd-El-Malek, Y., Monib, M. \& Hazem, A. Nature 198, 775-776 (1961).

3.

Crofts, T. S. et al. Nat. Chem. Biol. https://doi.org/10.1038/s41589-018-0052-1 (2018).

4.

Page, M. G. P. \& Bush, K. Curr. Opin. Pharmacol. 81, 91-97 (2014).

5

Llarull, X. X. et al. Curr. Opin. Microbiol. 13, 551-557 (2010).

6.

Rutledge, P. J. in 2-Oxoglutarate Dependent Oxygenases (eds Schofield, C. \& Hausinger, R.) 414-424 (Royal Society of Chemistry, Cambridge, 2015).

7.

Valle, F. et al. Trends Biochem. Sci. 16, 36-40 (1991).

8.

Huberts, D. H. \& van der Klei, I. J. Biochim. Biophys. Acta 1803, 520-525 (2010). 
